\title{
An Intramucosal Large Cell Neuroendocrine Carcinoma Arising Within a Tubulovillous Adenoma With High- Grade Dysplasia of the Rectosigmoid Colon Treated With Polypectomy
}

\author{
Youran Zou ${ }^{\mathrm{a}}$, Hong Shib ${ }^{\mathrm{b}}$, Meagan Costedio ${ }^{\mathrm{c}}$, Xiuli Liu ${ }^{\mathrm{a}, \mathrm{d}}$
}

\begin{abstract}
High-grade neuroendocrine carcinoma (NEC) has been previously reported to arise within preexisting adenoma but is usually at an advanced stage and carries a dismal prognosis and is almost always treated with surgical resection. Intramucosal NECs treated with polypectomy have not been reported. Herein, we report the first case of intramucosal NEC treated by polypectomy. An 87-year-old white male was referred to the Cleveland Clinic for a polyp detected in an outside hospital. A $40 \mathrm{~mm}$ sessile polyp was visualized in the rectosigmoid colon using flexible sigmoidoscopy. Polypectomy was performed and histopathology revealed multiple fragments of tubulovillous adenoma (TVA) with extensive high-grade dysplasia (HGD). Notably, there was a focus $(1 \mathrm{~mm})$ of NEC confined to lamina propria and intimately related to the adenomatous component. This focus of intramucosal NEC was confirmed by its strong and diffuse synaptophysin and chromogranin A immunoreactivity and near 100\% Ki-67 labeling index. Immunohistochemistry using a panel of antibodies against mismatch repair proteins was performed and both the adenomatous and NEC components had normal staining for MLH1, PMS2, MSH2, and MSH6. This, to the best of our knowledge, is the first case of intramucosal NEC treated with polypectomy reported to date. Patient was alive 1 year after polypectomy. Clinical behavior remains to be seen with long-term follow-ups.
\end{abstract}

Keywords: Colon; Cancer; High-grade neuroendocrine carcinoma; Polypectomy

Manuscript accepted for publication November 14, 2014

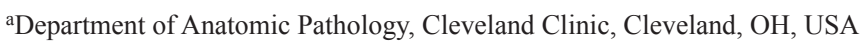
${ }^{b}$ Cardiovascular Research Institute, Case Western Reserve University, Cleveland, $\mathrm{OH}, \mathrm{USA}$

${ }^{\mathrm{c}}$ Gastroenterology, Digestive Disease Institute, Cleveland Clinic, Cleveland, $\mathrm{OH}, \mathrm{USA}$

${ }^{\mathrm{d} C o r r e s p o n d i n g ~ A u t h o r: ~ X i u l i ~ L i u, ~ C l e v e l a n d ~ C l i n i c ~ L e r n e r ~ C o l l e g e ~ o f ~ M e d i-~}$ cine of Case Western Reserve University, 9500 Euclid Avenue/L25, Cleveland, OH 44195, USA. Email: liux3@ccf.org

doi: http://dx.doi.org/10.14740/jmc1996w

\section{Introduction}

Neuroendocrine carcinoma (NEC) is an uncommon tumor in the large bowel and comprises about $0.6 \%$ of all carcinomas of the large intestine [1]. It can be divided into three categories including small cell carcinoma, large cell carcinoma and mixed adeno-neuroendocrine carcinoma (MANEC) according to the most recent WHO classification [1].

Previous reports have found that a proportion of NECs arise within preexisting adenomatous lesions [2-6]. However, the NECs of the large intestine reported so far, to the best of our knowledge, are advanced lesions that at least invade submucosa and are associated with early lymph node or liver metastasis and have much worse prognosis than adenocarcinoma [7,8]. Only one intramucosal NEC was reported in the literature, but it was treated with surgical resection and had no follow-up information [9]. Here we report a case of intramucosal NEC arising from a tubulovillous adenoma (TVA) with high-grade dysplasia (HGD) in the rectosigmoid colon treated with polypectomy.

\section{Case Report}

\section{Methods}

The polypectomy specimen was fixed in $10 \%$ neutral buffered formalin and processed in a usually fashion. Paraffin-embedded blocks were sectioned ( $5 \mu \mathrm{m}$ thick) and stained with hematoxylin and eosin. Immunohistochemical stains were performed as follows: synaptophysin (Clone Snp88; Biogenex), chromogranin A (Clone DAK-A3; Dako), Ki-67 (Clone 30-9; Ventana), TTF-1 (Clone 8G7G3/1; Ventana), CDX-2 (Clone CDX2-88; Biogenex) and $\beta$-catenin (Clone 14 BD; Bioscience). The intactness of mismatch repair machinery was assessed by immunohistochemical stains for MLH1 (Clone G168-15; Biocare), PMS2 (Clone A16-4; BD), MSH2 (Clone FE11; Biocare), and MSH6 (Clone BC/44; Biocare).

\section{Case history}

The patient was an 87-year-old white male who presented to 

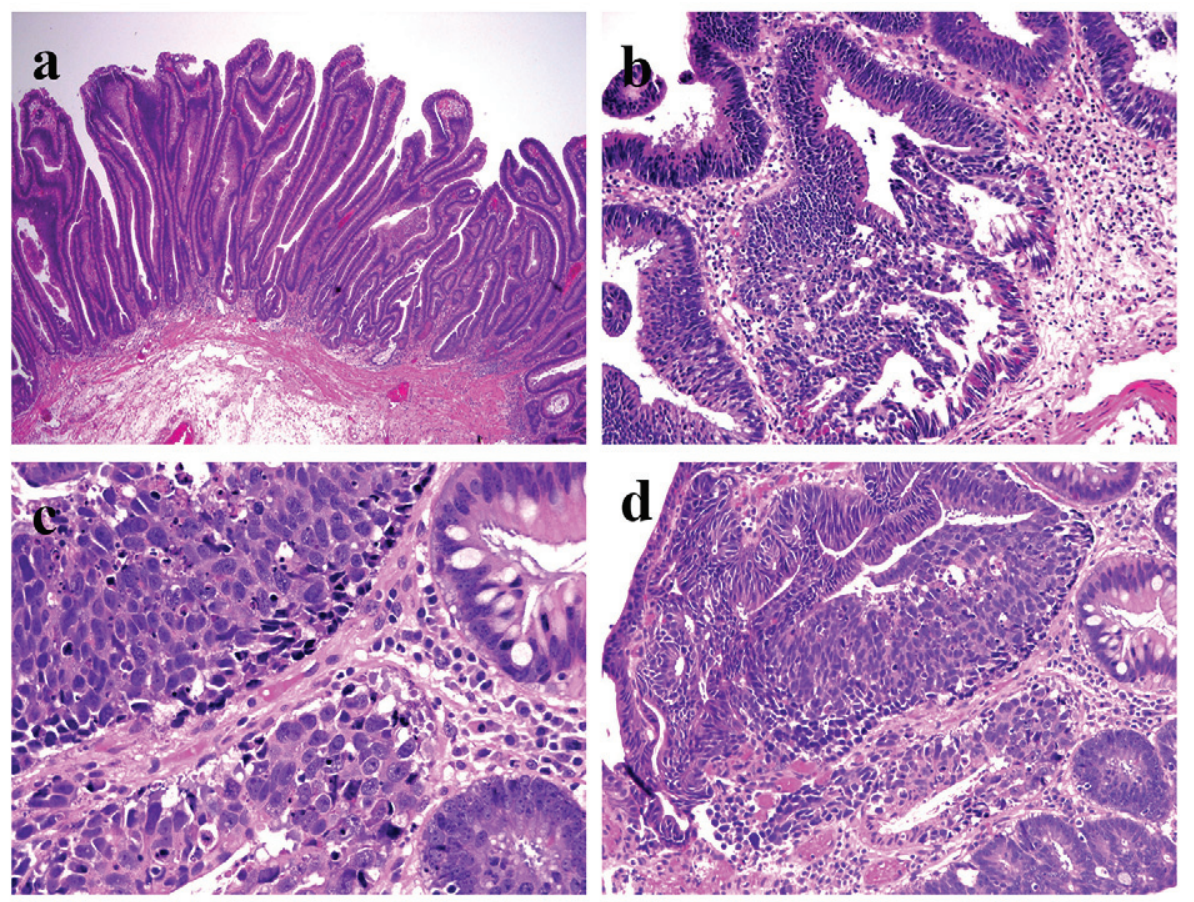

Figure 1. Histologic features of intramucosal NEC arising from a TVA with HGD. (a) Villous component of the adenoma (H\&E stain, $\times 40$ ); (b) HGD in the adenoma (H\&E stain, $\times 200$ ); (c) NEC in the lamina propria (H\&E stain, $\times 400$ ); (d) NEC with relation to adenoma $(\mathrm{H} \& \mathrm{E}, \times 200)$.

our hospital with previous colonoscopy and biopsy at an outside hospital about 3 months prior to this visit which showed a TVA with HGD. He had no bleeding, weight loss or changes in bowel movement patterns. He was a current smoker with more than 50 pack years of smoking. No family history of cancer was noted.

\section{Imaging/endoscopic findings}

Flexible sigmoidoscopy revealed an annular sessile polyp measuring $40 \mathrm{~mm}$ in greatest dimension and occupying $75 \%$ of the circumference in the rectosigmoid colon. The polyp was removed with a piecemeal technique using a hot snare. The polyp was felt to be completely removed endoscopically with the base cauterized. A computed tomography of the abdomen was performed after polypectomy and no evidence of metastatic disease was identified.

\section{Pathology}

Multiple fragments of TVA are seen (Fig. 1a). Areas of HGD characterized by architectural complexity and high cytological atypia (round and vesicular nuclei and prominent nucleoli) were identified (Fig. 1b). A few closely located foci (we deemed them as one lesion due to the piecemeal nature of the polypectomy) of high-grade NEC was noted in both the epithelium and subepithelial lamina propria (Fig. 1c). A hybrid gland consisting of both components was identified (Fig. 1d).
Within the NEC, the tumor cells had a high nucleus to cytoplasm (N/C) ratio. Nuclei were enlarged, oval to round and vesicular with focal nuclear molding. Half of the tumor cells demonstrated prominent nucleoli. A small to moderate amount of cytoplasm was noted in the tumor cells. Although obvious necrosis was not noted in the NEC, apoptosis was brisk (Fig. 1d). Mitosis was seen at a rate of $>20$ mitotic figures/10 HPFs. No angiolymphatic invasion was identified. Immunoreactivity for synaptophysin (diffuse and strong) and chromogranin A (focal) and high Ki-67 labeling index (near 100\%) confirmed a high-grade NEC (Fig. 2a-c). CDX-2 was diffusely positive in the adenomatous areas but negative in the NEC. Both adenomatous and NEC components showed cytoplasmic and focally nuclear immunoreactivity in addition to a membranous staining pattern for $\beta$-catenin (images not shown) and were negative for TTF-1 immunoreactivity. Immunohistochemical study for mismatch repair (MMR) proteins (MLH1, PMS2, MSH2 and MSH6) revealed normal expression of all four MMR proteins in the adenomatous and NEC nuclei.

\section{Discussion}

Neuroendocrine tumors (NETs) are the neoplastic counterpart of the neuroendocrine cells distributed throughout the human body. They comprise a group of neoplasm that share some distinct features such as organoid, nested or trabecular growth patterns, "salt and pepper" chromatin, positive staining with neuroendocrine markers and presence of ultrastructural neurosecretory granules. According to the most current WHO 


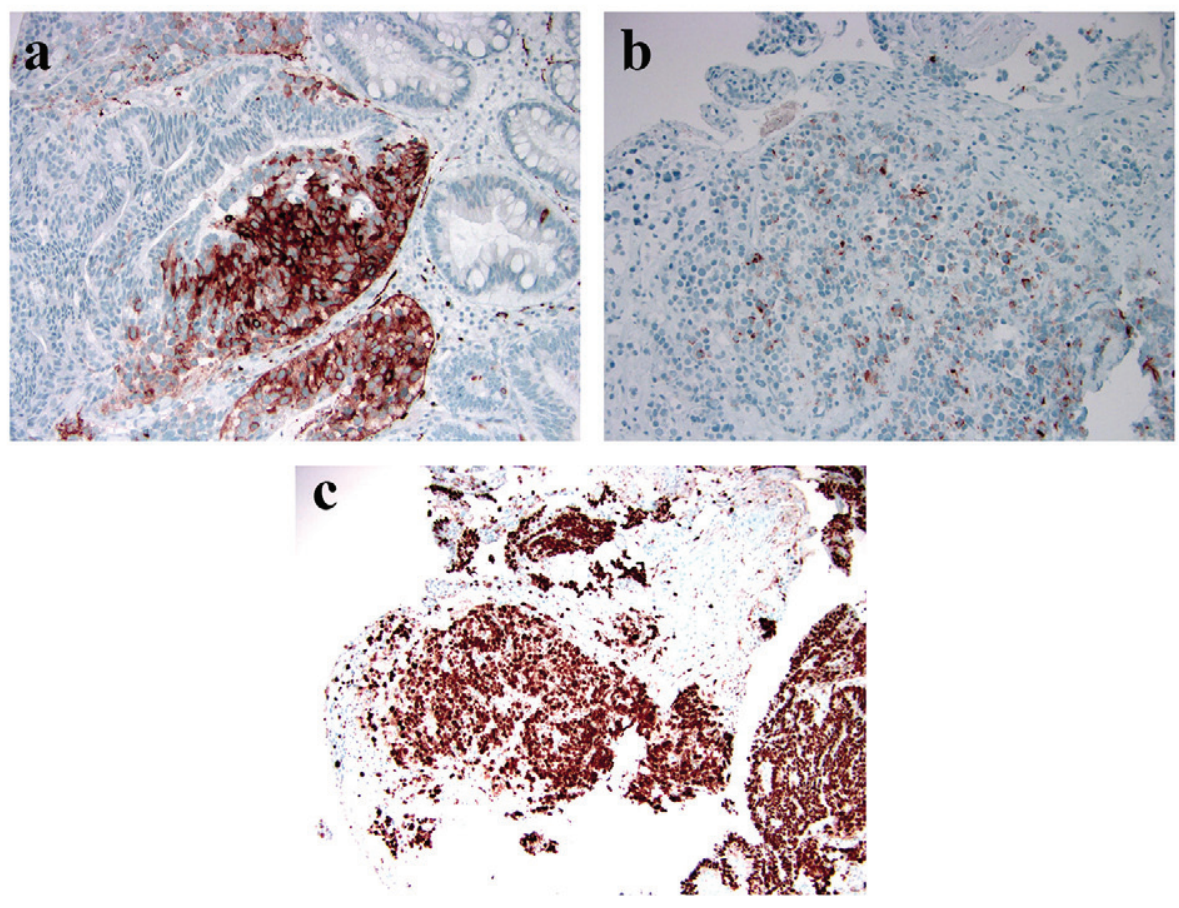

Figure 2. Immunophenotype of this high-grade NEC. (a) Strong and diffuse immunoreactivity of synaptophysin (peroxidasestain, $\times 100$ ); (b) Focal immunoreactivity of chromogranin with a perinuclear dot-like staining pattern (peroxidase stain, $\times 200$ ); (c) High Ki-67 labeling index (peroxidase stain, $\times 100$ ).

classification, NETs in the gastrointestinal tract are classified into three grades including well-differentiated (WD) NET, low grade (G1) WDNET, intermediate grade (G2) NET and poorly differentiated (high-grade) NEC (G3) [1]. The NEC is further split into small cell carcinoma, large cell carcinoma and MANEC based on morphology, which is largely analogous to their pulmonary counterparts $[1,10]$. Besides the nested or diffuse growth pattern and frequent mitosis and necrosis, small cell carcinomas typically have high $\mathrm{N} / \mathrm{C}$ ratio, fusiform molding nuclei without prominent nucleoli, little cytoplasm and prominent crush artifact, while large cell carcinomas are characterized by polygonal cells with abundant cytoplasm, round nuclei and prominent nucleoli. In our case, the tumor is best classified as large cell type since over half of the cells have round nuclei with prominent nucleoli, a small to moderate amount of cytoplasm, despite the presence of focal nuclear molding.

NEC arising in the colon was first described by Gould and Chejfec in 1978 [3]. The mean and median ages were 64 and 66 years and no sexually predilection was found $[4,5]$. The previously reported cases almost invariably behaved in an aggressive manner $[2,4,6]$. Many patients had either lymph node or liver metastasis at the time or presentation and most quickly succumbed to the disease. In one large series, the median survival time was 15.7 months and the 2-year and 5-year disease-specific survival rates were $25.4 \%$ and $14.8 \%$, respectively [6]. Due to its aggressive behavior, intramucosal NECs are extremely rare and only one has been previously reported. In that case, the tumor was removed by colectomy but there was no clinical follow-up information [9].
An association of NEC with adenomas has been reported previously [3]. This was further supported by additional reports. For example, Mills and workers identified five cases of small cell undifferentiated carcinoma of the colon with neuroendocrine features in which four cases were associated with an overlying adenoma with villous elements [4]; Burke et al reported 38 cases of small cell carcinoma of the colon and found that most occur in the right colon, and overlying adenomas were identified in $45 \%$ of cases [2]; and Shia et al found $31 \%(20 / 65)$ of NECs were associated with an adenomatous component [6]. The observation that NEC is frequently associated with overlying adenomas/adenocarcinomas suggests it might share similar oncogenic pathways with colorectal adenoma and adenocarcinoma. This concept has been further supported by two recent reports that demonstrated identical loss of heterozygosity, KRAS mutation, and BRAF/KRAS mutations in colonic NECs and their overlying adenoma or their accompanying adenocarcinoma $[7,11]$. The immediate proximity of the NEC in our cases to the adenomatous epithelium and the observed $\beta$-catenin nuclear translocation in both adenomatous and NEC components confirm the NEC is derived from the adenoma. These findings support that a substantial percent of NECs may be arising from the preexisting adenoma.

The NEC in our case appears to be confined to the lamina propria, which is a major difference from most of the previous cases which were invasive at the time of diagnosis. How to manage NEC at its earliest stage (i.e. intramucosal) is problematic. Although lymphatics have been proven to exist in the junction of lamina propria and muscularis mucosae [12], which renders a theoretical possibility for intramucosal carci- 
noma to metastasize, a study suggests that endoscopic intervention alone may be sufficient even for poorly differentiated intramucosal carcinoma [8]. Interestingly one of their cases has focal small cell differentiation but confirmation of its neuroendocrine nature was not attempted. Yet given the extraordinarily aggressive behavior of NEC, and limited case numbers reported to date, simply extrapolating that it would be cured after polypectomy may be imprudent. However, our patient was alive 1 year after polypectomy.

We hope our current case report will bring more similar cases with long-term follow-ups to the literature to help reveal the behavior of intramucosal high-grade NEC and determine whether polypectomy may be an adequate treatment.

\section{References}

1. Bosman FT, Carneiro F, Hruban RH. WHO Classification of Tumors of the Digestive System, 4th edition, 2010:174-177.

2. Burke AB, Shekitka KM, Sobin LH. Small cell carcinomas of the large intestine. Am J Clin Pathol. 1991;95(3):315321.

3. Gould VE, Chejfec G. Neuroendocrine carcinomas of the colon. Ultrastructural and biochemical evidence of their secretory function. Am J Surg Pathol. 1978;2(1):31-38.

4. Mills SE, Allen MS, Jr., Cohen AR. Small-cell undifferentiated carcinoma of the colon. A clinicopathological study of five cases and their association with colonic adenomas. Am J Surg Pathol. 1983;7(7):643-651.

5. Petrelli M, Tetangco E, Reid JD. Carcinoma of the colon with undifferentiated, carcinoid, and squamous cell features. Am J Clin Pathol. 1981;75(4):581-584.
6. Shia J, Tang LH, Weiser MR, Brenner B, Adsay NV, Stelow EB, Saltz LB, et al. Is nonsmall cell type highgrade neuroendocrine carcinoma of the tubular gastrointestinal tract a distinct disease entity? Am J Surg Pathol. 2008;32(5):719-731.

7. Huang J, Behrens C, Wistuba, II, Gazdar AF, Jagirdar J. Clonality of combined tumors. Arch Pathol Lab Med. 2002;126(4):437-441.

8. Lewin MR, Fenton H, Burkart AL, Sheridan T, Abu-Alfa AK, Montgomery EA. Poorly differentiated colorectal carcinoma with invasion restricted to lamina propria (intramucosal carcinoma): a follow-up study of 15 cases. Am J Surg Pathol. 2007;31(12):1882-1886.

9. Gaffey MJ, Mills SE, Lack EE. Neuroendocrine carcinoma of the colon and rectum. A clinicopathologic, ultrastructural, and immunohistochemical study of 24 cases. Am J Surg Pathol. 1990;14(11):1010-1023.

10. Travis WD, Travis WD, Colby TV, Corrin B, Shimosato Y, Brambilla E, and Collaborators from 14 Countries. World Health Organization Pathology Panel: World Health Organization. Histological Typing of Lung and Pleural Tumors. International Histological Classification of Tumors. Third Edition, Springer Verlag, Berlin, 1999.

11. Karkouche R, Bachet JB, Sandrini J, Mitry E, Penna C, Cote JF, Blons H, et al. Colorectal neuroendocrine carcinomas and adenocarcinomas share oncogenic pathways. A clinico-pathologic study of 12 cases. Eur J Gastroenterol Hepatol. 2012;24(12):1430-1437.

12. Fenoglio CM, Kaye GI, Lane N. Distribution of human colonic lymphatics in normal, hyperplastic, and adenomatous tissue. Its relationship to metastasis from small carcinomas in pedunculated adenomas, with two case reports. Gastroenterology. 1973;64(1):51-66. 